\title{
Surprising Mechanical and Thermodynamic Properties of the Non-Muscle Myosin Type II (NMMII) in Myofibroblasts
}

\author{
Yves Lecarpentier*1, Olivier Schussler ${ }^{2}$ and Alexandre Vallée ${ }^{3}$ \\ ${ }^{1}$ Centre de Recherche Clinique, Grand Hôpital de l'Est Francilien, Meaux, France \\ ${ }^{2}$ Department of Cardiovascular Surgery, centre hospitalo-universitaire de Genève, Switzerland
}

${ }^{3}$ Department Hypertension and Cardiovascular Prevention Unit, Hôtel-Dieu, AP-HP Paris France

*Corresponding author: Yves Lecarpentier, Centre de Recherche Clinique, Grand Hôpital de l’Est Francilien, 77104 Meaux, France

\section{ARTICLE INFO}

Received: 慧 February 02, 2019

Published: 閩 February 22, 2019

Citation: Yves L, Olivier S, Alexandre V. A Surprising Mechanical and Thermodynamic Properties of the Non-Muscle Myosin Type II (NMMII) in Myofibroblasts. Biomed J Sci \& Tech Res 15(1)2019. BJSTR. MS.ID.002635.

Keywords: Non-Muscle Myosin; Myofibroblast; Statistical Mechanics; Placenta; Mesenchymal Stem Cell; Entropy Production Rate
ABSTRACT

Non-muscle contractile tissues are now recognized. They differ from muscle contractile tissues by their basal cell, the myofibroblast and their molecular motor, the non-muscle myosin type II (NMMII). Surprisingly, muscles and non-muscles share several fundamental contractile properties, namely the Frank-Starling phenomenon and the hyperbolic relationship of A. V. Hill between tension and maximum shortening velocity. They quantitatively diverge from muscles by their mechanical properties which are much slower and develop less tension than muscles. This also applies to ultraslow kinetics of the non-muscle myosin (NMMII) compared to those of the muscle myosin (MMI and MMII). Both muscles and non-muscles also share common properties of statistical mechanics: they behave near equilibrium and in a linear stationary regime. Entropy production rate is much lower in non-muscles than in muscles due to their low thermodynamic flow. Interestingly, the order of magnitude of both the force generated by one actin-myosin interaction and the thermodynamic efficiency of this interaction is the same in the two systems. Human placental stem villi and mesenchymal stem cells from human bone marrow in the presence of TGF-beta are largely composed of myofibroblasts and represent two kinds of non-muscle contractile tissues. This leads to a classification of contractile systems which can be subdivided into two classes, i. e., muscle tissues and non-muscle tissues.

\section{Opinion}

Mechanical and thermodynamic properties of striated (skeletal and cardiac) and smooth muscles have been described in an exhaustive manner for a long time. These contractile systems comply with Frank-Starling's law [1, 2], which specifies that the initial length of a muscle fiber is determined by its preload and that muscle active tension increases with the preload level. Moreover, A. V. Hill, established a hyperbolic relationship between the isotonic tension ( $\mathrm{T}$ ) level and the maximum shortening velocity (V) $[3,4]$ whose curvature is related to the thermodynamic properties of the muscle system. The higher the curvature, the more economical the muscular system is. This hyperbolic T-V relationship is crucial because it is necessary to be able to apply the A. F. Huxley phenomenological theory of muscle contraction [5]. This allows to determine the molecular properties of actin-myosin crossbridges (CBs) namely: the unitary force generated by one CB interaction, the number of active CBs per volume unit of tissue, the attachment and detachment CB kinetics and the maximum myosin ATPase activity [6]. The basic cell of muscle systems is the myocyte, and its molecular motor is the type I and II muscle myosin (MI and MII). In our laboratory, we developed the formalism of statistical mechanics by means of the grand canonical ensemble to calculate numerous thermodynamic parameters such as statistical entropy characterizing the dispersal of energy, internal energy, chemical affinity, thermodynamic flow (TFl), thermodynamic force (TFo), and entropy production rate (diS/dt) which is the product of TFl and TFo [6-9]. 
Open living contractile systems exchange energy and matter with the surroundings. In statistical mechanics, the grand canonical ensemble can be applied to complex open systems such as muscle contractile tissues. Statistical mechanics combined with A. Huxley's equations provide a powerful tool for showing the link between the chemo-mechanical properties of CB molecular motors and their thermodynamic characteristics. This led us to show that striated and smooth muscles behave near from equilibrium i. e, chemical affinity $\ll$ RT (R: gas constant; T: Kelvin temperature), and in a linear stationary state, i. e., that TFl linearly varies with TFo. Under the linear regime wherein the Onsager phenomenological laws [10] can be used, a near equilibrium system can tend towards a linear stationary regime $[7,9]$.

Thus, diS/dt can reach a minimum level that represents the criterion of stability of a stationary state. The irreversibility of chemical processes is quantified by diS/dt. The higher the diS/dt, the further the chemical system moves far away from equilibrium [7-9]. We have determined the CB molecular properties by means of A. F. Huxley equations, applied statistical mechanics to various muscles and established that muscles behaved near equilibrium and in a linear stationary state $[6,11,12]$. In addition to muscle systems, other tissues also exhibit a contractile behavior. One of them is human placental stem villi, in which cells resembling smooth muscle cells have been observed since the early twentieth century $[13,14]$. Similarily to that observed in muscle tissues, placental stem villi are able to contract after potassium chloride stimulation or by applying an electrical field [15-17]. We have studied the mechanical and thermodynamic properties of the human placenta by using the same techniques as those for muscles. To our surprise, we have observed that the Frank-Starling phenomenon is also present in the human placental stem villi [18]. This strongly suggests that the ultrastructure of human placental stem villi is relatively highly organized to allow the sliding of actin filaments along myosin filaments as classically reported in both striated and smooth muscles. The T-V hyperbolic relationship is also observed in human placental stem villi [19].

This made it possible to determine their molecular characteristics and thermodynamic properties [20] as we had done previously in muscles. The fundamental difference between muscles and placenta is that shortening velocity of placenta is much slower than that of muscles and placental stem villi develop a lower tension than muscles do. In addition, kinetics of actinmyosin CBs and myosin ATPase activity are much lower in placenta than those observed in muscles [12,21]. Importantly, the unitary force generated by one actin-myosin $\mathrm{CB}$ interaction and the $\mathrm{CB}$ thermodynamic efficiency are of the same order of magnitude in both muscles and non-muscle contractile placental stem villi. In placenta, the basic contractile cell is the myofibroblast [22] and the molecular motor is the non-muscle myosin type II (NMMII) [23]. From a thermodynamic viewpoint, the human placenta also operates near equilibrium and in a linear stationary regime, but closer from equilibrium than muscles are $[12,20]$. In placenta, entropy production rate is dramatically low due to the low TFl.

We have recently shown that mesenchymal stem cells (MSCs) from human bone marrow collected after a femoral neck fracture present the same mechanical properties as those of human placenta stem villi when MSCs are included in collagen scaffolds in the presence of TGF-beta [24]. Under these conditions, MSCs differentiate into myofibroblasts expressing NMMIIA. The mechanical properties observed in collagen scaffolds are similar to those of the placenta and of the same order of magnitude in terms of shortening velocity and tension. Myofibroblasts play a key role in tissue repair processes such as skin healing. That is how they have been discovered by Gabbiani et al. [25]. The canonical WNT/beta-catenin pathway promotes the differentiation of MSCs and fibroblasts into myofibroblasts. Canonical WNT and TGF-beta pathways promote tissue fibrosis in the heart, lungs, liver and kidneys . In addition, myofibroblasts also play a key role in many cancers $[26,27]$.

In conclusion, muscles and non-muscle contractile systems share four fundamental properties. First, stimulation of contractile tissues can be induced by either an electric field or potassium chloride. Second, they present the Starling phenomenon. Third, they exhibit a hyperbolic tension-velocity relationship. Fourth, they can relax spontaneously by inhibiting the CB cycle by means of either 2,3-butanedione monoxime or blebbistatin which inhibit the CB cycle and by activating the NO-cGMP pathway by means of either isosorbide dinitrate or sildenafil [18]. The NMMII molecular motor of non-muscle contractile tissues exhibits ultraslow CB kinetics. However, the unitary $\mathrm{CB}$ force and efficiency of the non-muscle myosin (NMMII) in myofibroblasts from human placenta are similar in magnitude to those of MMI and II in myocytes of striated and smooth muscle cells. Both non-muscle contractile systems operate near from equilibrium and in linear stationary regime. We propose to subdivide contractile tissues into two classes according to their molecular mechanical CB properties and CB statistical mechanics, i.e., muscle myocyte tissues expressing MM1 and II and non-muscle myofibroblast tissues expressing NMMII.

\section{References}

1. Frank O (1895) Zur Dynamik des Herzmuskels. Z Biol 32: 273-277.

2. Starling EH (1918) The Linacre Lecture on the Law of the Heart. Longmans, Green \& Co, London.

3. Hill AV (1938) The heat of shortening and the dynamic constants of muscle. Proc R Soc Lond Biol Sci 126: 136-195.

4. Hill AV (1951) Thermodynamics of muscle. Nature 167(4245): 377-380.

5. Huxley AF (1957) Muscle structure and theories of contraction. Prog Biophys Biophys Chem 7: 255-318.

6. Lecarpentier Y, Blanc FX, Quillard J, Hebert JL, Krokidis X, et al. (2005) Statistical mechanics of myosin molecular motors in skeletal muscles. J Theor Biol 235(3): 381-392.

7. Prigogine I (1967) Introduction to thermodynamics of Irreversible Processes, Wiley J, New York, USA. 
8. Prigogine I, Nicolis G, Babloyantz A (1974) Nonequilibrium problems in biological phenomena. Annals of the New York Academy of Sciences 231(1): 99-105.

9. Kondepudi D, Prigogine I (1999) Modern thermodynamics from heat engines to dissipative structures, Wiley \& Sons, New York, USA.

10. Onsager L (1931) Reciprocal relations in irreversible processes II. Phys Rev 38: 405-426.

11. Lecarpentier Y, Krokidis X, Martin P, Pineau T, Hebert JL, et al. (2008) Increased entropy production in diaphragm muscle of PPAR alpha knockout mice. J Theor Biol 250(1): 92-102.

12. Lecarpentier Y, Claes V, Krokidis X, Vallée A (2017) Comparative Statistical Mechanics of Muscle and Non-Muscle Contractile Systems Stationary States of Near-Equilibrium Systems in A Linear Regime. Entropy Journal 19(10): 558.

13. Iizuka S (1916) Uber Verkammen von Muskelfasern in der menschlichen Placenta. Beitr Geburtsh Gynaek 19: 101.

14. Dubreuil G, Rivière M (1932) Formations fibromusculaires du chorion et villosités du placenta humain. CR Soc Biol 111: 170-172.

15. Krantz KE, Panos TC, Evans J (1962) Physiology of maternalfetal relationship through the extracorporeal circulation of the human placenta. American journal of obstetrics and gynecology 83: 1214-1228.

16. King TM, Groeschel Stewart U (1965) Placental Contractile Protein American journal of obstetrics and gynecology 93: 253-258.

17. Farley AE, Graham CH, Smith GN (2004) Contractile properties of human placental anchoring villi, American journal of physiology. Regulatory. integrative and comparative physiology 287(3): R680-685.

18. Lecarpentier E, Claes V, Timbely O, Hebert JL, Arsalane A, et al. (2013) Role of both actin-myosin cross bridges and NO-cGMP pathway modulators in the contraction and relaxation of human placental stem villi. Placenta 34(12): 1163-1169.

\section{ISSN: 2574-1241}

DOI: 10.26717/BJSTR.2019.15.002635

Yves Lecarpentier. Biomed J Sci \& Tech Res

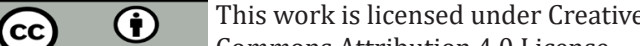

This work is licensed under Creative
Commons Attribution 4.0 License

Submission Link: https://biomedres.us/submit-manuscript.php
19. Lecarpentier Y, Claes V, Lecarpentier E, Guerin C, Hebert JL, et al. (2014) Ultraslow myosin molecular motors of placental contractile stem villi in humans. PloS one 9(9): e108814.

20. Lecarpentier Y, Claes V, Hebert JL, Krokidis X, Blanc FX, et al. (2015) Statistical Mechanics of the Human Placenta: A Stationary State of a NearEquilibrium System in a Linear Regime. PloS one 10(11): e0142471.

21. Kovacs M, Wang F, Hu A, Zhang Y, Sellers JR (2003) Functional divergence of human cytoplasmic myosin II: kinetic characterization of the nonmuscle IIA isoform. The Journal of biological chemistry 278(40): 38132 38140 .

22. Feller AC, Schneider H, Schmidt D, Parwaresch MR (1985) Myofibroblast as a major cellular constituent of villous stroma in human placenta. Placenta 6(5): 405-415.

23. Matsumura S, Sakurai K, Shinomiya T, Fujitani N, Key K, et al. (2011) Biochemical and immunohistochemical characterization of the isoforms of myosin and actin in human placenta. Placenta 32(5): 347-355.

24. Lecarpentier Y, Schussler O, Sakic A, Rincon Garriz JM, Soulie P, et al. (2018) Human Bone Marrow Contains Mesenchymal Stromal Stem Cells That Differentiate In Vitro into Contractile Myofibroblasts Controlling T Lymphocyte Proliferation. Stem Cells Int 2018: 6134787.

25. Gabbiani G, Hirschel BJ, Ryan GB, Statkov PR, Majno G (1972) Granulation tissue as a contractile organ. A study of structure and function. The Journal of experimental medicine 135(4): 719-734.

26. Bochaton Piallat ML, Gabbiani G, Hinz B (2016) The myofibroblast in wound healing and fibrosis: answered and unanswered questions F1000Research 26: 5 .

27. Lecarpentier Y, Schussler O, Claes V, Vallée A (2017) The Myofibroblast: TGF $\beta-1$, A Conductor which Plays a Key Role in Fibrosis by Regulating the Balance between PPAR $\gamma$ and the Canonical WNT Pathway. Nuclear Receptor Research p. 4.

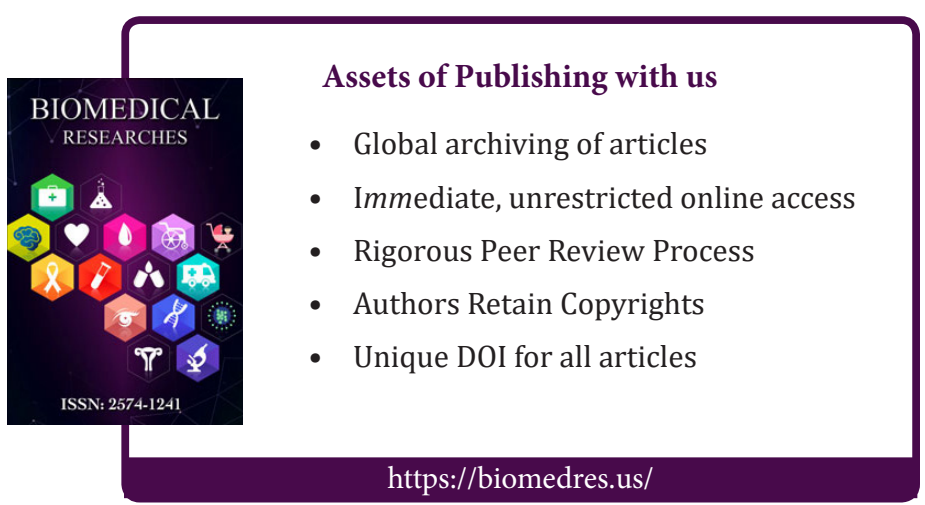

